\title{
Unsteady near-shore natural convection induced by surface cooling
}

\author{
YADAN MAO†, CHENGWANG LEI \\ AND JOHN C. PATTERSON§ \\ School of Engineering and Physical Sciences, James Cook University, Townsville QLD 4811, Australia
}

(Received 22 March 2009; revised 25 August 2009; accepted 26 August 2009;

first published online 4 December 2009)

Natural convection in calm near-shore waters induced by daytime heating or nighttime cooling plays a significant role in cross-shore exchanges with significant biological and environmental implications. Having previously reported an improved scaling analysis on the daytime radiation-induced natural convection, the authors present in this paper a detailed scaling analysis quantifying the flow properties at varying offshore distances induced by nighttime surface cooling. Two critical functions of offshore distance have been derived to identify the distinctness and the stability of the thermal boundary layer. Two flow scenarios are possible depending on the bottom slope. For the relatively large slope scenario, three flow regimes are possible, which are discussed in detail. For each flow regime, all the possible distinctive subregions are identified. Two different sets of scaling incorporating the offshore-distance dependency have been derived for the conduction-dominated region and stable-convection-dominated region respectively. It is found that the scaling for flow in the stable-convectiondominated region also applies to the time-averaged mean flow in the unstable region. The present scaling results are verified by numerical simulations.

Key words: boundary layers, gravity currents, Navier-Stokes equations

\section{Introduction}

The intrinsic nature of a near-shore topography - an increasing water depth in the offshore direction - implies that, when subject to the same rate of daytime heating or nighttime cooling, the depth averaged local temperature will decrease or increase respectively in the offshore direction. This generates a horizontal temperature gradient that drives an onshore-offshore exchange flow, often referred to as a 'thermal siphon'.

The significance of this buoyancy-driven flow has been demonstrated by field measurements (Adams \& Wells 1984; Monismith, Imberger \& Morison 1990). It is revealed that the thermal siphon is a predominant, yet often overlooked, mechanism driving the cross-shore circulation in calm near-shore regions with limited winddriven and tidal circulation. More recently, increasing attention has been paid to the biological implications of this 'thermal siphon'. The field experiment of Monismith et al. (2006) suggests that the 'thermal siphon' may be a general feature of hydrodynamic processes of reefs and the costal ocean, helping to alleviate the stress of coral bleaching as well as enhancing connectivity between the reef and the ocean

$\dagger$ Email address for correspondence: yadan.mao@jcu.edu.au

$\ddagger$, Present address: School of Civil Engineering, The University of Sydney, NSW 2006, Australia 
(Monismith 2007). The investigation of Niemann et al. (2004) on the flow of near-reef phytoplankton off the Gulf of Aqaba reveals that the gravity currents induced by surface cooling are a dominant force in driving the cross-shore circulation, linking the coastal ecosystem with the adjacent ocean by exporting phytoplankton and other suspended matter to the deep waters of the Red Sea. In addition to these biological effects, this 'thermal siphon' also has a significant environmental impact, exchanging terrestrially derived pollutions or nutrients in the form of particles or solutes in the littoral region with the open ocean or lake. In this sense, the strength of this siphon is directly relevant to the resilience of the near-shore water to pollution, which in turn affects the eutrophic state of the near-shore water body, governing the population of algae and other phytoplankton.

Efforts in quantifying the strength of the thermal siphon have also been made through asymptotic solutions, scaling analysis, numerical simulations and laboratory experiments. For the daytime heating case, the asymptotic solutions of Farrow \& Patterson (1994) and the scaling by Lei \& Patterson (2002) provide important insight into natural convection induced by absorption of solar radiation. More recently, the scaling analysis by Mao, Lei \& Patterson (2009a) extended the scaling of Lei \& Patterson (2002), revealing the variation of thermal flow with offshore distance through two position-dependent critical functions and two sets of position-dependent scalings. For the nighttime surface cooling case, Horsch \& Stefan (1988) conducted numerical simulations and laboratory experiments and found an approximately proportional relation between the flow rate and $R a^{1 / n}$, where $2<n<3$, for $R a$ in the range of $10^{4}$ $10^{8}$. The steady-state flow generated by surface cooling was reinvestigated by Sturman, Oldham \& Ivey (1999) through scaling analysis and laboratory measurements, which provided scales for horizontal exchange and flushing time with respect to the surface heat flux and the inclination angle of the bottom slope. More recently, Lei \& Patterson (2005) provided more detailed scaling than the previous investigations of natural convection induced by surface cooling, classifying the flow into three regimes, namely a conductive, a transitional and a convective regime. Later numerical simulations and laboratory experiments of Bednarz, Lei \& Patterson $(2008,2009)$ focus qualitatively on the development of sinking plumes with time in the unstable flow regime in the presence of surface cooling. The results of Bednarz et al. $(2008,2009)$ verified the scaling for the onset time of instability proposed by Lei \& Patterson (2005).

The above investigations shed light on near-shore natural convection induced by surface cooling. However, they did not capture or quantify the variation of flow properties with offshore distance, which is essential for deeper insight into the cooling phase of the diurnal 'thermal siphon'. The variation of velocity with offshore distance is crucial to ascertain the detailed rate of flow exchange and transport of particles and solutes at varying offshore distance, and the variation of temperature and the dominant mode of heat transfer with offshore distance has important implications on the distribution of temperature-sensitive biological organisms, such as coral reefs.

The variation of flow quantities with offshore distance and the resultant flow scenarios and flow regimes have been revealed for the daytime heating case through scaling analysis by the present authors (Mao et al. 2009a). In that case, the incoming radiation is absorbed in an exponentially decreasing manner from the surface, resulting in a stable stratification of the upper region of the water column. In shallow waters where the water depth is less than the attenuation depth of the solar radiation, the residual radiation which reaches the bottom is absorbed by the bottom surface. The simple model adopted for this process by Farrow \& Patterson (1994) is that this absorbed radiation is re-emitted as a boundary heat flux. This results in a potentially unstable thermal boundary layer along the bottom slope, in which the 
layer characteristics vary with offshore distance. The absorption of the exponentially decaying radiation with water depth by the water body results in a stable stratification near the water surface. The competing mechanisms of the stable stratification and the position-dependant potentially unstable thermal boundary layer were explored by Mao et al. (2009a), in which distinct subregions of the flow were identified, including conductive, stable convective and unstable convective regions.

Significantly, different mechanisms exist in the nighttime cooling case. As opposed to the non-uniform heat flux (exponentially decaying with water depth) on the sloping bottom caused by absorption of radiation for the daytime heating case, cooling is assumed to be introduced through a uniform heat flux at the water surface, resulting in a potentially unstable surface thermal boundary layer, which may result in sinking plumes. In the shallow regions, the flow is expected to be dominated by thermal conduction, whereas in the deeper regions the flow may be dominated by the sinking plumes and an underlying circulation generated by the cross-shore temperature gradient. Apart from the different heat flux conditions, the stable stratification due to the absorption of radiation by the water body in the daytime heating case is not relevant to the cooling case. Further, compared with the heating phase of the diurnal cycle, an additional mechanism is present for the cooling case, that is, the Phillips mechanism (Phillips 1970; Wunsch 1970), which is discussed in detail in Lei \& Patterson (2005).

The horizontal temperature gradient in this investigation results from the nearshore geography. In a wider context, a horizontal temperature gradient can also be directly imposed by non-uniform thermal forcing at the horizontal boundaries, such as in the experiment of Rossby (1965) and Mullarney, Griffiths \& Hughes (2004). A recent review of horizontal convection generated by this type of thermal forcing can be found in Hughes \& Griffiths (2008).

Similar to the previous scaling analysis on the radiation-induced natural convection, the present investigation is aimed at quantifying the variation of flow properties across shore and identifying all possible flow scenarios, flow regimes and distinct flow subregions for natural convection induced by surface cooling, extending the scaling analysis of Lei \& Patterson (2005). The results of the present scaling analysis are verified by numerical simulations.

\section{Model formulation}

A two-dimensional wedge model with a horizontal water surface representing the offshore direction captures the essential geometric features generating the driving mechanism of the 'thermal siphon'. This model has been adopted as a basic model in many theoretical investigations and numerical simulations of near-shore buoyancydriven flows (Horsch \& Stefan 1988; Farrow \& Patterson 1993a,b, 1994; Sturman et al. 1999; Lei \& Patterson 2002, 2005; Mao, Lei \& Patterson 2007, 2009b; Mao et al. 2009a), and is again adopted here as sketched in figure 1. A bottom slope of $A$, corresponding to a maximum water depth of $h$ and a horizontal length of $L$, parameterizes the model. With the Bousinesq assumption, the Navier-Stokes and energy equations governing the flow and temperature evolution within the wedge are

$$
\begin{gathered}
u_{x}+v_{y}=0, \\
u_{t}+u u_{x}+v u_{y}=-\rho_{0}^{-1} p_{x}+v \nabla^{2} u, \\
v_{t}+u v_{x}+v v_{y}=-\rho_{0}^{-1} p_{y}+v \nabla^{2} v+g \beta\left(T-T_{0}\right), \\
T_{t}+u T_{x}+v T_{y}=k \nabla^{2} T,
\end{gathered}
$$




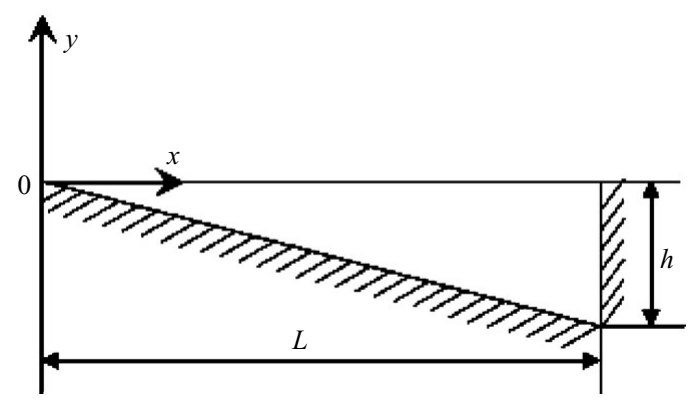

FIGURE 1. Geometry of the flow domain.

where $u$ and $v$ are the velocity components in the horizontal and vertical directions, respectively; $x$ and $y$ are the horizontal and vertical coordinates originating from the tip; $T$ is the fluid temperature; and $p$ is the pressure. The parameters $\rho_{0}, k, v$ and $\beta$ are respectively the density, thermal diffusivity, kinematic viscosity and thermal expansion coefficient of the fluid at a reference temperature $T_{0}$.

In the present model, the water in the wedge is initially stationary and isothermal (at the reference temperature $T_{0}$ ). The endwall and the bottom slope are rigid, non-slip $(u=v=0)$ and adiabatic, whereas the water surface is stress free $\left(u_{y}=0\right.$ and $\left.v=0\right)$. The cooling is introduced with a constant heat flux through the water surface as

$$
\frac{\partial T}{\partial y}=-\frac{1}{k}\left(\frac{I_{0}}{\rho_{0} C_{p}}\right)=-\frac{H_{0}}{k}, \quad y=0,
$$

where $I_{0}$ is the imposed surface heat flux and $C_{p}$ is the specific heat.

Similar to the daytime heating case reported in Mao et al. (2009a), the endwall has no relevance to the following scaling and it is only assumed for the numerical simulations so that simulations can be carried out in a finite domain. For later verification of the scaling using simulation results, flow properties from the simulations are selected far from the endwall to avoid its effects, as discussed in Mao et al. (2009a).

\section{Scaling analysis}

\subsection{Initiation of the flow}

As soon as cooling is initiated at the water surface, a thermal boundary layer begins to grow beneath the water surface. This thermal layer has an adverse temperature gradient with warmer fluid underlying cooler fluid, and thus is potentially unstable. In contrast to radiation-induced natural convection, for which the dominant mechanism is a heat flux at the sloping bottom, there are two independent mechanisms for natural convection induced by surface cooling, namely, the Phillips mechanism (Phillips 1970; Wunsch 1970) and unequal heat loss. The Phillips mechanism induces a flow along the sloping boundary as a consequence of the curvature of the isotherms necessary to meet the condition of thermal insulation. Lei \& Patterson (2005) compared the flows driven by these two mechanisms and concluded that for $t>t_{m} \sim R a_{h}^{-1 / 2} h^{2} / k$, the unequal heat loss dominates over the Phillips mechanism, where the Rayleigh number $R a_{h}$ is defined as $R a_{h}=g \beta H_{0} h^{4} / \nu \kappa^{2}$. Assuming $t_{m}$ to be small at relatively large Rayleigh numbers, the Phillips mechanism is neglected in the later stage of the scaling of Lei \& Patterson (2005). Based on this result, we focus on the mechanism of unequal heat loss in the following scaling. 
The thermal boundary layer starts to grow through conduction as soon as cooling is initiated. A balance between the unsteady term and the diffusion term in the energy equation (4) yields a scale for the thickness of the thermal boundary layer:

$$
\delta_{T} \sim(k t)^{1 / 2},
$$

within which the temperature decrease due to the surface heat flux specified in (5) can be expressed as

$$
T_{S} \sim H_{0} k^{-1 / 2} t^{1 / 2} .
$$

As the bottom slope is assumed to be small in order to represent field situations, the vertical velocity down the bottom slope is negligible compared to the horizontal velocity. Therefore, a balance between the pressure term and the buoyancy term in the vertical momentum equation (3) yields the scale for the pressure $p$ :

$$
p \sim g \beta \rho_{0} T_{S} \delta_{T} \sim g \beta \rho_{0} H_{0} t .
$$

Within the horizontal momentum equation (2), comparison of the unsteady inertia term $O(u / t)$, the advection term $O\left(u^{2} / x\right)$ over an arbitrary length scale $x$ and the viscous term $O\left(v u / \delta_{T}^{2}\right)$ yields that the viscous term dominates among the three terms for $\operatorname{Pr}>1$ and $u t / x<P r$, where $P r$ is the Prandtl number defined as

$$
\operatorname{Pr}=v / k \text {. }
$$

For $\operatorname{Pr}>1$, such as the water case considered here, at the early stage when $u t<\operatorname{Pr} x$, the balance in the horizontal momentum equation is between the viscous term and the buoyancy induced pressure gradient, which yields a velocity scale of

$$
u \sim g \beta H_{0} \frac{k t^{2}}{v x} \sim R a \frac{t^{2} k^{3}}{L^{4} x},
$$

where $R a$ is the global Rayleigh number defined as

$$
R a=g \beta H_{0} L^{4} /\left(v k^{2}\right) .
$$

It appears in (10) that the velocity scale depends on both the domain length $L$ and the offshore location $x$. However, the dependence on the domain length $L$ is included only because of the presence of the Rayleigh number, which is defined in terms of the domain length $L$. The purpose of introducing the Rayleigh number here is to facilitate subsequent verification by numerical simulations which are conducted in a finite domain. Since the thermal forcing is imposed over the horizontal length $L$, to set the present work in a wider context of horizontal convection problems (refer to the recent review of Hughes \& Griffiths 2008), the Rayleigh number here is defined in terms of the horizontal length $L$ rather than the maximum water depth $h$ as in previous investigations (Horsch \& Stefan 1988; Lei \& Patterson 2002, 2005; Mao et al. 2009a,b). Related by the bottom slope $A$, both $L$ and $h$ are characteristic length scales, and $R a$ and $R a_{h}$ differ by a factor of $A^{4}$, which needs to be taken into account for comparison between the present and the previous scaling results.

It is worth noting that a variable length scale $x$ is adopted in the scaling reported above, whereas a fixed length scale $L$ is used in Lei \& Patterson (2005). It will soon become clear that a variable length scale and the analysis based upon it reveal more detailed features of the thermal flow than a fixed length scale.

\subsection{Steady state of the boundary layer}

While heat is conducted away from the thermal boundary layer through the surface heat flux, heat is also convected into the thermal boundary layer since convection 
brings warmer water towards the shore. Initially, heat conducted away from the boundary layer is greater than that convected into it, and hence the thermal boundary layer continues to grow. As the flow velocity increases with time (see (10)), so does convection. Once the heat convected into the boundary layer balances that conducted away, the thermal boundary layer stops growing and the flow becomes steady. The balance between convection and conduction in energy equation (4) can be expressed as

$$
\frac{u T}{x} \sim \frac{k T}{\delta_{T}^{2}} .
$$

This balance yields the time scale for the steady state of the thermal boundary layer at location $x$ :

$$
t_{c} \sim x^{2 / 3} R a^{-1 / 3} \frac{L^{4 / 3}}{k} .
$$

After accounting for the difference in the Rayleigh number definitions, scale (13) can also be obtained by replacing the fixed length scale in $t_{c}$ derived by Lei \& Patterson (2005) with a variable length scale $x$.

On the other hand, a comparison between the thickness of the thermal boundary layer (6) and the local water depth $A x$ results in the time scale for the thermal boundary layer to reach the sloping bottom:

$$
t_{d} \sim \frac{A^{2} x^{2}}{k} .
$$

Depending on the local water depth, there are two possibilities: (i) $t_{d}<t_{c}$; the thermal boundary layer reaches the bottom slope before convection becomes significant and thus the thermal boundary layer is indistinct, encompassing the entire local water depth. (ii) $t_{d}>t_{c}$; in this case, convection becomes significant before the thermal boundary layer reaches the bottom slope, and thus the thermal boundary layer stops growing and remains distinct. Therefore, the criterion for a distinct thermal boundary layer is $t_{d}>t_{c}$ that is equivalent to

$$
R a>A^{-6} x^{-4} L^{4} .
$$

The right side of (15) is a function of the horizontal position $x$, and is denoted by $f_{1}(x)$ as

$$
f_{1}(x) \sim A^{-6} x^{-4} L^{4} .
$$

From (15), the dividing position $x_{0}$ that marks the switch from indistinct thermal boundary layer to a distinct thermal boundary layer as offshore distance increases is scaled as

$$
x_{0} \sim R a^{-1 / 4} A^{-3 / 2} L .
$$

It is clear from (17) that as the Rayleigh number increases, the region with distinct thermal boundary layer expands towards the shore.

For an indistinct thermal boundary layer, substituting (14) into (6), (7) and (10), the steady state scales for the thickness of the thermal boundary layer, the temperature and the velocity are obtained as

$$
\begin{gathered}
\delta_{T} \sim A x, \\
T_{S} \sim A x H_{0} / k, \\
u \sim R a A^{4} L^{-4} x^{3} k,
\end{gathered}
$$


and the volumetric flow rate across a sectional plane at the steady state is given by

$$
Q \sim u \delta_{T} \sim \operatorname{Ra} A^{5}(x / L)^{4} k .
$$

For a distinct thermal boundary layer, substituting (13) into (6), (7) and (10) yields the steady state scales for the thickness of thermal boundary layer, the temperature and the velocity respectively:

$$
\begin{gathered}
\delta_{T} \sim R a^{-1 / 6} L^{2 / 3} x^{1 / 3}, \\
T_{S} \sim R a^{-1 / 6} L^{2 / 3} x^{1 / 3} H_{0} k^{-1}, \\
u \sim R a^{1 / 3} L^{-4 / 3} x^{1 / 3} k .
\end{gathered}
$$

From (22) and (24), it can be obtained that the volumetric flow rate at steady state for a distinct thermal boundary layer is

$$
Q \sim u \delta_{T} \sim R a^{1 / 6}(x / L)^{2 / 3} k
$$

The criterion for a distinct thermal boundary layer can be alternatively derived by comparing (22) with the local depth $A x$.

\subsection{Onset of instability}

The adverse temperature gradient near the water surface is a potential source of instability. Although describing the detailed dynamics of an unstable or turbulent boundary layer is beyond the capability of the present scaling, the scaling analysis is able to provide a criterion for instability to occur which results in the scales for the onset time of instability and the horizontal extent of the unstable region. A RayleighBénard type instability sets in if the local temperature gradient in the thermal boundary layer exceeds a certain critical value, which is embodied by $R a_{L}>R a_{c}$, where $R a_{c} \approx 657.5$ based on a free-free boundary configuration (Drazin \& Reid 1981 ), and $R a_{L}$ is the local Rayleigh number of the thermal boundary layer defined as

$$
R a_{L} \sim \frac{g \beta T_{S} \delta_{T}^{3}}{\nu k} \sim R a\left(\frac{t}{L^{2} / k}\right)^{2} .
$$

Therefore, instability, if present, sets in at the time scale

$$
t_{B} \sim\left(\frac{R a_{c}}{R a}\right)^{1 / 2} \frac{L^{2}}{k},
$$

so long as $t_{B}<t_{c}$.

Although $R a_{L}$ increases with time, there is an upper limit for $R a_{L}$. When the flow reaches its steady state at $t_{c}$, both $T_{S}$ and $\delta_{T}$ reach their maximum values and thus the maximum $R a_{L}$ is given by $R a_{L}\left(t_{c}\right)$. If $R a_{L}\left(t_{c}\right)<R a_{c}$, then the flow is always stable. In other words, instability, if present, has to occur before $t_{c}$. Therefore, the criterion for instability to happen can be derived in two equivalent ways (i) $R a_{L}\left(t_{c}\right)>R a_{c}$ or (ii) $t_{c}>t_{B}$. Both lead to the same condition:

$$
R a>R a_{c}^{3}(L / x)^{4} .
$$

The right side of (28) is also a function of the horizontal position $x$, and is denoted by $f_{2}(x)$ as

$$
f_{2}(x) \sim R a_{c}^{3}(L / x)^{4}
$$



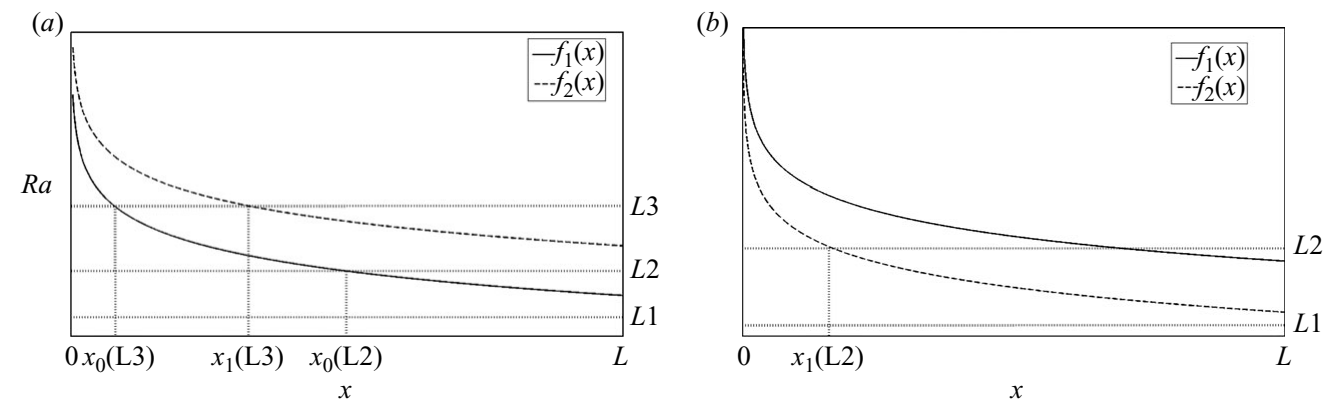

FIgURE 2. Profiles of $f_{1}(x)$ and $f_{2}(x)$ for $(a) A>R a_{c}^{-1 / 2}$ (b) $A<R a_{c}^{-1 / 2}$. Horizontal dotted lines represent typical flow regimes.

From (28), the dividing position $x_{1}$ that marks the switch from stable region near shore to unstable region offshore is scaled as

$$
x_{1} \sim L R a_{c}^{3 / 4} R a^{-1 / 4} .
$$

It is clear that as $R a$ increases, the dividing position $x_{1}$ decreases, indicating the expansion of unstable region towards the shore.

The critical Rayleigh number $R a_{c}$ appears as a constant in (27) and (30). Similar to the case of radiation-induced natural convection (Mao et al. 2009a), a slight variation of the exact value of $R a_{c}$ from the assumed value does not affect the scaling results.

Comparing the above scaling results, including two critical functions, $f_{1}(x)$ and $f_{2}(x)$, and two sets of scaling, (18)-(21) and (22)-(25), with their counterparts for radiation-induced natural convection in Mao et al. (2009a) (as mentioned in §3.1, a factor of $A^{4}$ needs to be taken into account due to the difference in the definitions of the Rayleigh number), it is noticeable that the results are similar except that all the exponential terms appearing in the results of radiation-induced natural convection disappear in the present results for natural convection induced by surface cooling. As mentioned in the introduction, the exponentially decaying absorption of radiation with water depth results in an exponential term in the heat flux at the sloping bottom, which is carried throughout the scaling for radiation-induced natural convection.

\subsection{Possible flow regimes}

So far two criteria have been derived for characterizing the boundary layer flow locally: (i) the thermal boundary layer is distinct if $R a>f_{1}(x)$; (ii) the flow is unstable if $R a>f_{2}(x)$. Both $f_{1}(x)$ and $f_{2}(x)$ decrease with offshore distance $x$. Comparison between $f_{1}(x)$ and $f_{2}(x)$ yields two possible scenarios as shown in figure 2. Each of these scenarios consists of several distinct flow regimes depending on the global Rayleigh number. Typical flow regimes for each scenario are represented by the horizontal dotted lines in figure 2.

Differently from the radiation-induced natural convection, which has four different scenarios (Mao et al. 2009a), the flow induced by surface cooling has only two scenarios: $(a)$ for large bottom slopes $A>R a_{c}^{-1 / 2}$ (figure $2 a$ ); and (b) for small bottom slopes $A<R a_{c}^{-1 / 2}$ (figure $2 b$ ). The two additional scenarios for the radiationinduced natural convection result from comparison of the maximum water depth with an additional key parameter intrinsic to the radiation problem - the penetration depth of radiation, which is not relevant to the cooling case. 


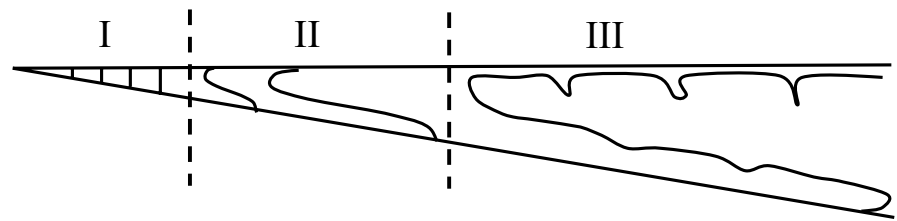

FIGURE 3. Sketches of expected isotherms for different flow subregions: region I $R a<f_{1}(x)$ indistinct, conductive; Region II $f_{1}(x)<R a<f_{2}(x)$, distinct, stable convective; Region III $R a>f_{2}(x)$, unstable convective.

The following discussion will mainly focus on scenario $(a)$ with a brief discussion of scenario $(b)$. A detailed discussion of scenario $(b)$ can be made following the same procedures outlined for scenario $(a)$ :

(a) $A>R a_{c}^{-1 / 2}$. In this case $f_{1}(x)<f_{2}(x)$ for all $x$. As $0<x \leqslant L$, both $f_{1}(x)$ and $f_{2}(x)$ reach their minimum at $x=L$. The two minimum values, $A^{-6}$ and $R a_{c}^{3}$, determine three possible flow regimes depending on the global Rayleigh number. Similar to natural convection induced by absorption of radiation (Mao et al. 2009a), under certain conditions, the entire flow region may consist of three subregions with distinct flow features illustrated by the isotherms sketched in figure 3. More detailed features of these subregions are described below and will be verified by numerical simulations. For a given Rayleigh number, the entire domain may be composed of one, two or all three of the subregions, as described below:

(i) $R a<A^{-6}$, represented by $L 1$ in figure $2(a)$. In this flow regime with $R a<f_{1}(x)<f_{2}(x)$ for all $x$, the thermal boundary layer is indistinct and the flow is stable. The thermal boundary layer encompasses the entire domain before convection becomes significant. Therefore, the entire flow domain is conductive, corresponding to region I in figure 3.

(ii) $A^{-6}<R a<R a_{c}^{3}$, represented by $L 2$ in figure $2(a)$. In this flow regime, $R a<f_{2}(x)$ for all $x$, and therefore the flow is stable over the entire domain. There is an intersection $x_{0}(L 2)$ between $R a$ and $f_{1}(x)$, which is scaled as (17). For $x<x_{0}(L 2), R a<f_{1}(x)$, the thermal boundary layer is indistinct and heat transfer is conduction-dominated. For $x>x_{0}(L 2), R a>f_{1}(x)$, the thermal boundary layer is distinct and the dominant mode of heat transfer switches to convection, corresponding to region II in figure 3. The flow remains stable and may be described as stable convection. Detailed discussion by Mao et al. (2009a) about the transfer from region I to region II for the radiative heating case can be also applied to the cooling case here.

(iii) $R a>R a_{c}^{3}$, represented by $L 3$ in figure 2(a). In this flow regime, $R a$ intersects with both $f_{1}(x)$ and $f_{2}(x)$ at $x_{0}(L 3)$ and $x_{1}(L 3)$ respectively, which are scaled by (17) and (30) respectively. The entire flow domain can be divided into three subregions by these two intersections, corresponding to all the subregions in figure 3. For $x<x_{0}(L 3), R a<f_{1}(x)<f_{2}(x)$, heat transfer is conductiondominated and the thermal boundary layer is indistinct at steady state. For $x_{0}(L 3)<x<x_{1}(L 3), \quad f_{1}(x)<R a<f_{2}(x)$, heat transfer is dominated by stable convection and the thermal boundary layer is distinct at steady state. For $x>x_{1}(L 3), f_{1}(x)<f_{2}(x)<R a$, the flow becomes unstable with instability setting in at time $t_{B}$, and both unstable plumes and the general convective circulation are present in this region.

(b) $A<R a_{c}^{-1 / 2}$. In this case, $f_{1}(x)>f_{2}(x)$ for all $x$ (figure $2 b$ ). The critical Rayleigh number for instability to occur is smaller than that for the presence of a distinct 
thermal boundary layer. For $R a>f_{2}(L) \sim R a_{c}^{3}$, instability occurs in the region of $x>x_{1}(L 2)$, where $x_{1}$ is scaled by (30). Once instability occurs, the growth of the thermal boundary layer is disturbed and no longer follows scale (6), and therefore $f_{1}(x)$ becomes irrelevant. As a result, there are only two flow regimes that scaling can predict, stable and unstable, represented respectively by $L 1$ and $L 2$ in figure $2(b)$.

\section{Numerical procedures}

\subsection{Governing equations}

Similar to the case of radiation heating (Mao et al. 2009a), a quasi-steady state can be reached for the case of surface cooling. At the quasi-steady state, since the surface heat flux is constant and all of the other boundaries are adiabatic, the temperature decreases at the same rate everywhere, and thus the difference between the local and the average temperatures becomes steady. With this quasi-steady assumption and the following normalization, equations (1)-(4) can be simplified into a new set of nondimensional governing equations containing the temperature difference, following the procedures outlined in Lei \& Patterson (2005):

$$
\begin{gathered}
u_{t}+u u_{x}+v u_{y}=-(\operatorname{Pr} \operatorname{Ra}) p_{x}+\operatorname{Pr} \nabla^{2} u \\
v_{t}+u v_{x}+v v_{y}=-(\operatorname{Pr} \operatorname{Ra}) p_{y}+\operatorname{Pr} \nabla^{2} v+(\operatorname{Pr} \operatorname{Ra}) \tau, \\
\tau_{t}+u \tau_{x}+v \tau_{y}=\nabla^{2} \tau+2, \\
u_{x}+v_{y}=0 .
\end{gathered}
$$

All the quantities above have been normalized by the following scales: $x, y \sim L$; $t \sim L^{2} / k ; \tau \sim H_{0} L / k ; u, v \sim k / L ; p_{x}, p_{y} \sim \rho_{0} g \beta H_{0} L / k$.

\subsection{Numerical method}

The governing equations (31)-(34) along with the specified boundary and initial conditions are solved numerically using a finite volume method. The SIMPLE scheme is adopted for pressure--velocity coupling and the QUICK scheme is applied for spatial derivatives. A second-order implicit scheme is applied for time discretization in calculating the transient flow.

The simulation is conducted in a triangular domain of a dimensionless depth of $h=0.1$, a dimensionless length of $L=1$, corresponding to a bottom slope of $A=0.1$, with a Prandtl number of $P r=7$. This parametric setting is relevant to Scenario (a). A mesh and time dependency test has been conducted using four different meshes, $125 \times 70,188 \times 105,251 \times 140$ and $313 \times 175$ for $R a=3.5 \times 10^{11}$, which is in the unstable regime and is the highest among all the simulated cases. To avoid singularity at the tip, a very small tip region $(x<0.02)$ was cut off and an extra vertical adiabatic wall was assumed. The cut off region accounts for only $0.04 \%$ of the entire domain and thus no significant modification to the flow is expected except at the very beginning of the flow development, which will be discussed below. All the meshes are distributed in a way so that the density of the mesh increases towards the boundaries. The time step is adjusted for the different meshes so that the CourantFriedrichs-Lewy (CFL) number remains approximately the same for different meshes. Figure 4 shows the time series of the maximum negative horizontal velocity obtained for the specified case with the four different meshes. Since this flow is in the unstable convection regime, the flow shows considerable fluctuations; however the standard deviations of the time series over a time period of 0.001 from the coarsest to the finest meshes are $2109,1769,1223$ and 1177 , converging to a constant as the mesh is refined. 


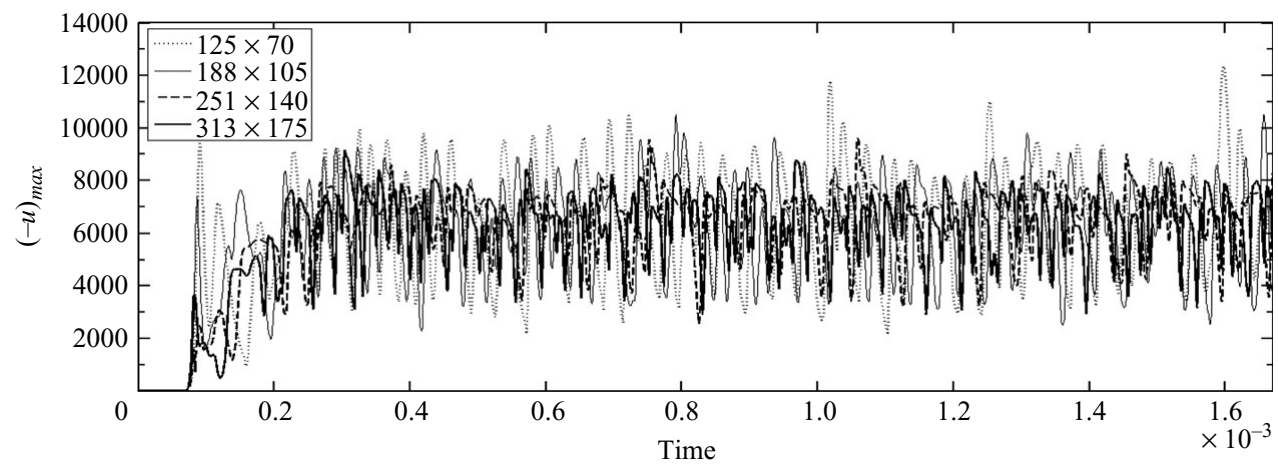

FIGURE 4. Time histories of the maximum negative horizontal component of velocity in the surface boundary layer along the vertical line of $x=0.35$ for $R a=3.5 \times 10^{11}$.

In order to ensure the accuracy of the solutions while keeping the calculation time manageable, the mesh of $251 \times 140$ with a time step of $4.0 \times 10^{-7}$ has been selected after the mesh and time-step dependency test.

Similar discussions to those of Mao et al. (2009a) can be made on the transition of the flow status from the initial to transitional and finally to quasi-steady state. A major difference in the simulated flow development between radiation-induced and surface-cooling-induced natural convection lies in the initial stage. Differently from the radiation-induced flow, the velocity of which starts to increase with time during the initial stage as soon as the thermal forcing is imposed, the flow induced by surface cooling (figure 4) shows no motion for a noticeable initial period $\left(\approx 7 \times 10^{-5}\right)$ with an approximately zero maximum flow velocity. This feature can be attributed to the growth of the thermal boundary layer and the truncation of the tip region. For the surface cooling case, a horizontal temperature gradient can only be established after the horizontal thermal boundary layer grows beyond the depth of the cutoff region, before which the flow remains stagnant. More discussion of this effect can be found in Lei \& Patterson (2005).

\section{Verification of scaling analysis}

The introduction of a variable offshore distance enables the present scaling analysis to reveal the variation of flow features with offshore distance not revealed in the previous scaling analysis of Lei \& Patterson (2005): the existence of distinct subregions and the respective scaling for them embodying the offshore-distance dependency. This section will focus on verifying these newly revealed flow properties using the results of the numerical simulations.

Scaling analysis has revealed that the dominant mode of heat transfer varies with distinct subregions. To validate this, horizontal heat transfer rates by conduction and convection are calculated from the simulation results. The total horizontal heat transfer rate, including the contributions of both conduction and convection, is averaged over the local water depth and defined in a dimensionless form as

$$
H(x)=\frac{1}{A x} \int_{-A x}^{0}\left(u \tau-\tau_{x}\right) \mathrm{d} y .
$$

The simulations involve a wide range of Rayleigh numbers to cover all the three possible flow regimes. All the Rayleigh numbers used in the simulations are shown in table 1 and plotted in figure 5 along with the profiles of $f_{1}(x)$ and $f_{2}(x)$. 
Flow regime

(i) $R a<A^{-6}$

(ii) $A^{-6}<R a<R a_{c}^{3}$

(iii) $R a>R a_{c}^{3}$

$R a$

$7 \times 10^{5}$

$2.1 \times 10^{7}, 3.5 \times 10^{7}, 5.6 \times 10^{7}, 7.0 \times 10^{7}, 8.4 \times 10^{7}, 1.4 \times 10^{8}, 2.1 \times 10^{8}$

$1.4 \times 10^{10}, 2.1 \times 10^{10}, 3.5 \times 10^{10}, 7.0 \times 10^{10}, 2.1 \times 10^{11}, 3.5 \times 10^{11}$

TABLE 1. Various Rayleigh numbers adopted in the numerical validation.

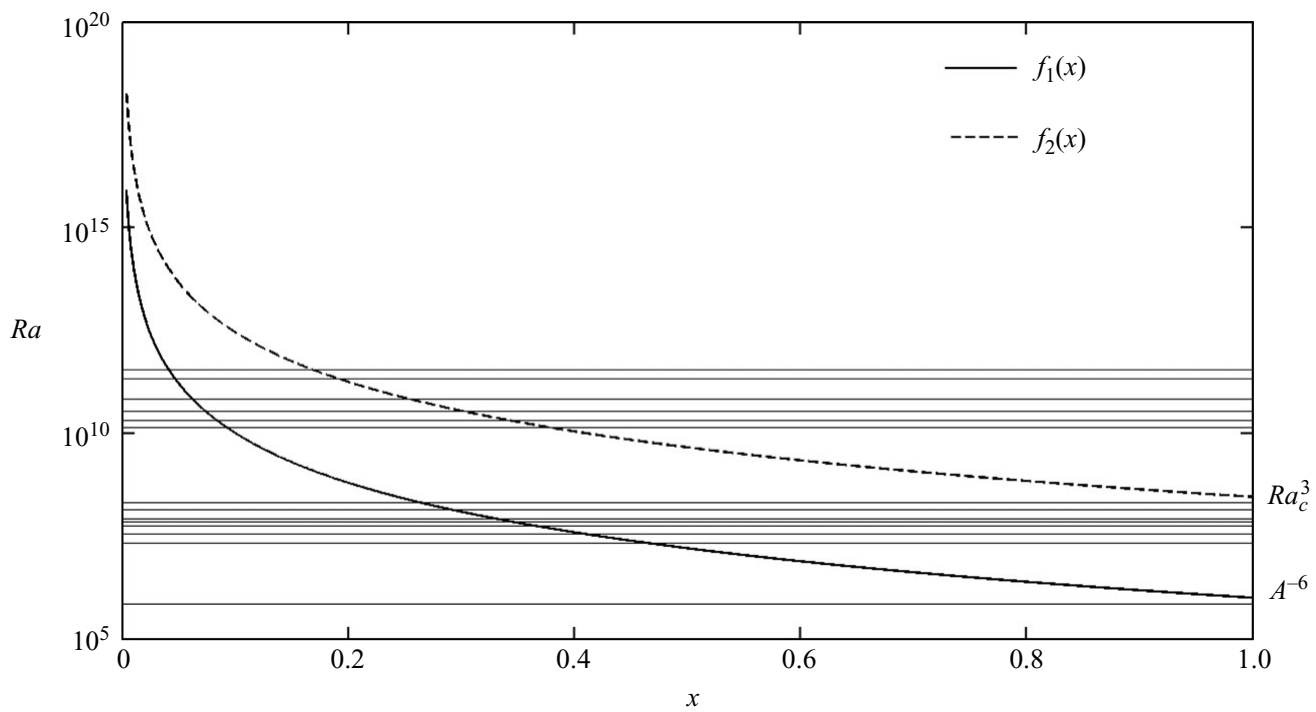

FIGURE 5. Typical profiles of $f_{1}(x)$ and $f_{2}(x)$ for scenario $(a)$ plotted with parameter values used in the numerical simulations; horizontal lines represent $R a$ values used in the numerical simulations. The minimum values of $f_{1}(x)$ and $f_{2}(x)$ are $A^{-6}$ and $R a_{c}^{3}$ respectively.

\subsection{Flow scenarios in different flow regimes}

\subsubsection{Regime $R a<A^{-6}$}

For the conductive regime $R a<A^{-6}$, figure 6 shows the simulation results at $R a=7 \times 10^{5}$. The nearly vertical isotherms in figure $6(a)$ confirm the presence of an indistinct thermal boundary layer. An anticlockwise flow is formed as shown in figure $6(b)$. The dominance of conduction in horizontal heat transfer is confirmed in figure $6(c)$. As the offshore direction is the assumed positive direction in our calculation, the negative sign of the heat transfer rate plotted in figure $6(c)$ indicates that heat is transferred towards the shore, which is contrary to the daytime heating case.

\subsubsection{Regime $A^{-6}<R a<R a_{c}^{3}$}

For the stable convective regime $A^{-6}<R a<R a_{c}^{3}$, figure 7 shows the simulation results for two Rayleigh numbers of $R a=2.1 \times 10^{7}$ and $2.1 \times 10^{8}$ respectively. Here, the vertical isotherms near the shore gradually transfer into curved isotherms with increasing curvature as offshore distance increases, indicating an increasing effect of convection in horizontal heat transfer. Figures $7(c)$ and $7(d)$ confirm that the dominant mode of horizontal heat transfer switches from conduction into stable convection as offshore distance increases. The horizontal position where conduction 
(a)

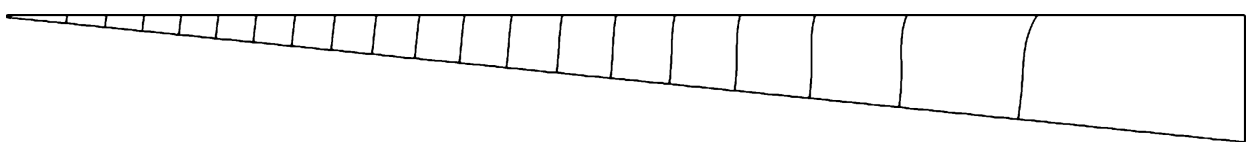

(b)

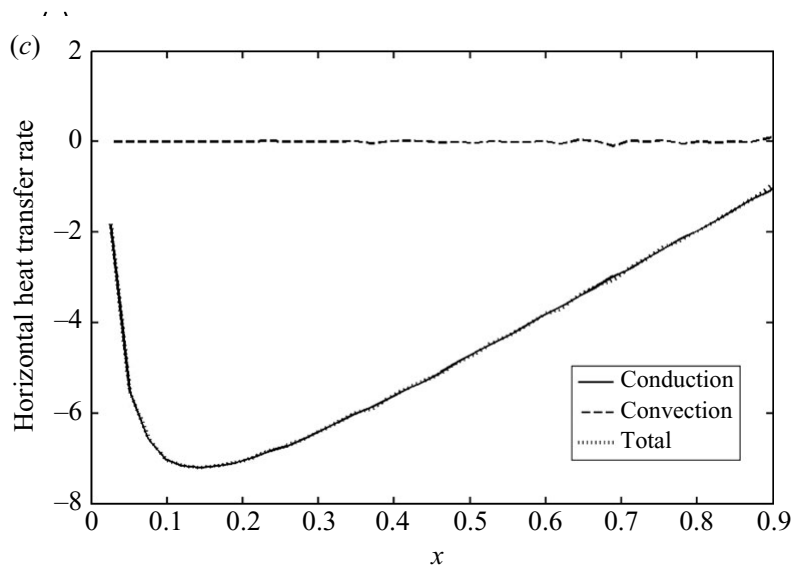

FigURE 6. Flow properties in the conductive regime of $R a<A^{-6}$. (a) Isotherms at an interval of 0.19. (b) Anticlockwise streamlines at an interval of 0.035. (c) Horizontal heat transfer rate averaged over the local water depth. The curve of the total heat transfer rate overlaps with the curve of heat transfer rate by conduction.

(a)

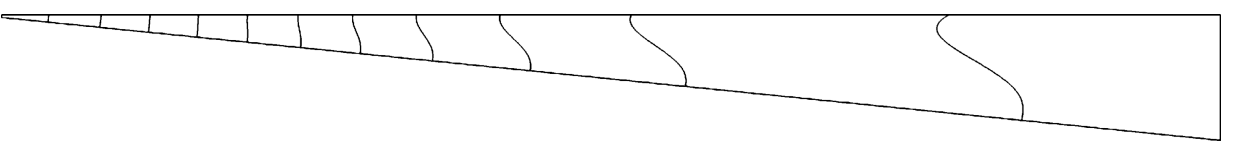

(b)

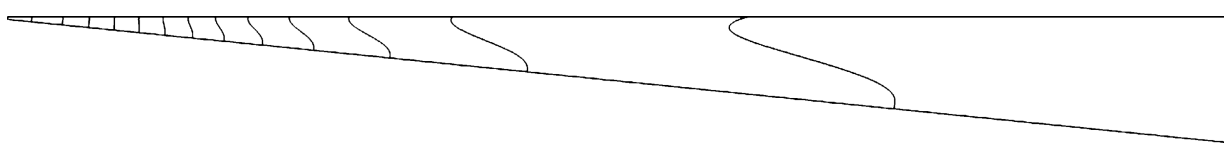

(c)

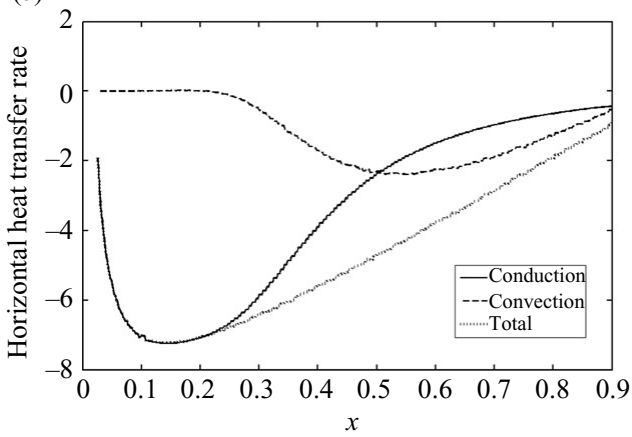

(d)

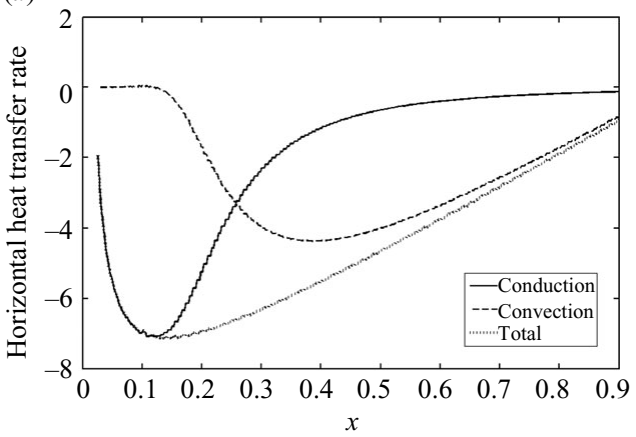

FiguRE 7. Flow properties in the convective regime of $A^{-6}<R a<R a_{c}^{3}$. (a) Isotherms for $R a=2.1 \times 10^{7}$ with an interval of 0.1407 . (b) Isotherms for $R a=2.1 \times 10^{8}$ with an interval of 0.0704. Profiles of the horizontal heat transfer rate averaged over the local water depth for (c) $R a=2.1 \times 10^{7}$ and (d) $R a=2.1 \times 10^{8}$. 


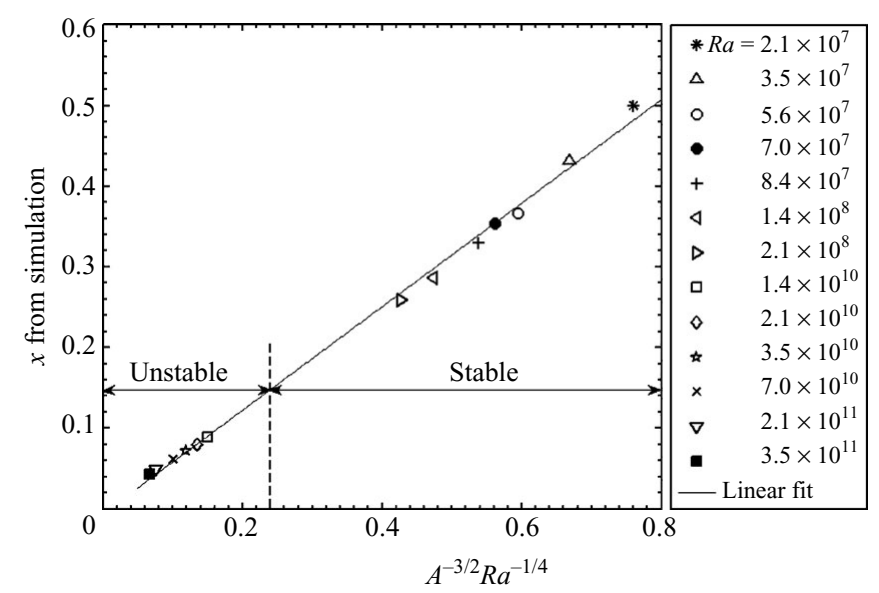

FIGURE 8. Dividing positions between conduction- and convection-dominated regions from numerical simulations versus scaling predictions. The dashed line approximately indicates the dividing position corresponding to the critical Rayleigh number for the switch from stable to unstable flow regime from the scaling analysis.

equals convection marks the dividing position between conduction- and convectiondominated regions. A comparison between figures $7(c)$ and $7(d)$ shows that as the Rayleigh number increases, the convection-dominated region expands towards the shore. The dividing positions obtained from simulations for different Rayleigh numbers are plotted against the scaling prediction of $x_{0} \sim A^{-3 / 2} R a^{-1 / 4} L$ in figure 8 . It is noted that the results obtained for the unstable flow regime of $R a>R a_{c}^{3}$ (see $\S 5.1 .3$ ), which also embodies a dividing position between conduction- and convectiondominated subregions, are also included in this plot. The clear linear correlation between the numerical data and the scaling prediction demonstrates that the dividing position (between conduction- and convection-dominated subregions) is well predicted by the scaling analysis.

\subsubsection{Regime $R a>R a_{c}^{3}$}

For the unstable convective regime $R a>R a_{c}^{3}$, figure 9 shows the isotherms, streamlines and horizontal heat transfer rates at the quasi-steady state. As the offshore distance increases, the isotherms (figures $9 a$ and $9 b$ ) transfer from vertical lines into curved lines and finally into wavy lines, indicating that the dominant mode of horizontal heat transfer changes from conduction into stable convection and finally into unstable convection, which is confirmed in figures $9(e)$ and $9(f)$. Comparisons of the results in figure 9 show that as the Rayleigh number increases, the unstable region expands towards the shore and the intensity of sinking plumes also increases. The clockwise flow near the endwall in figures $9(c)$ and $9(d)$ is caused by the endwall effect.

The positions where the wavy feature originates divide the stable and unstable subregions. To ascertain the dividing position, the standard deviation of the time series of the integrated horizontal convection over the local depth calculated over a time period of 0.0012 at the quasi-steady state is plotted against the offshore distance for different Rayleigh numbers in figure 10(a). It is expected that the standard deviation of the calculated convection heat transfer rate increases with offshore distance. However, owing to the endwall effect, the standard deviation decreases near the endwall. Near the tip region, the positions where the standard deviation exceeds 
(a)

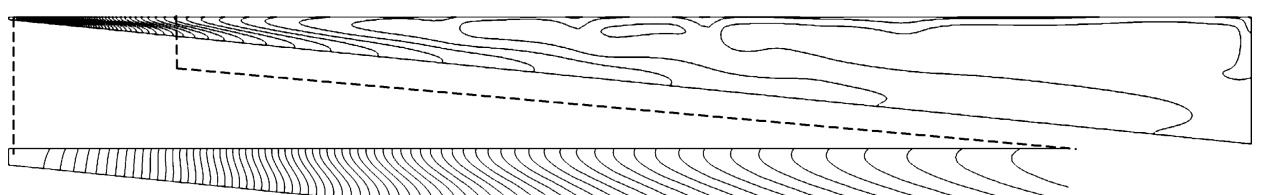

(b)

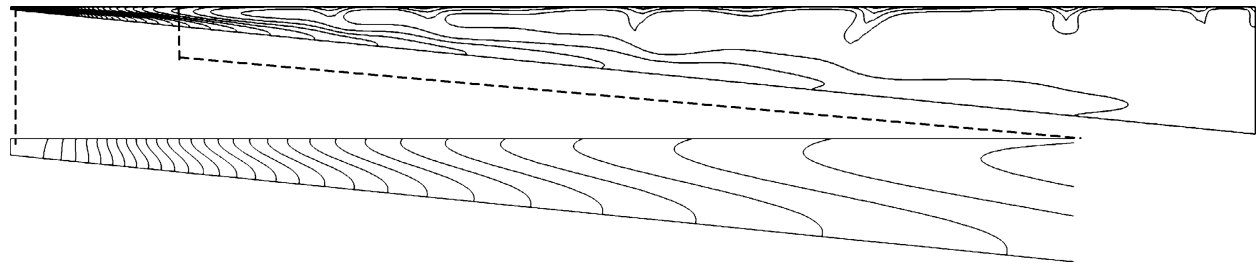

(c)

(d)

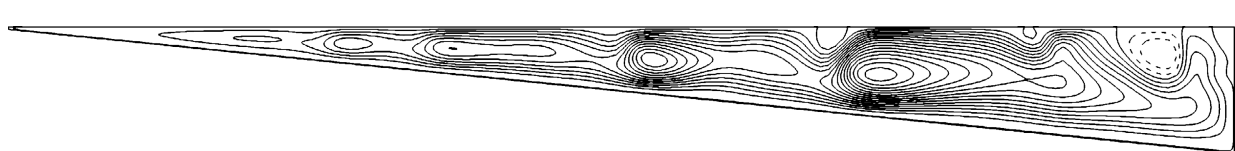

(e)

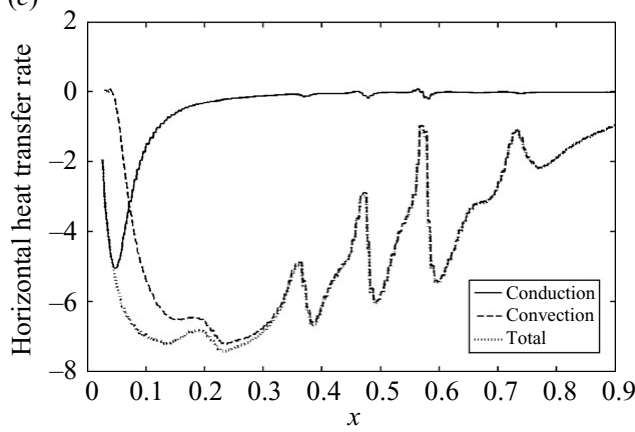

$(f)$

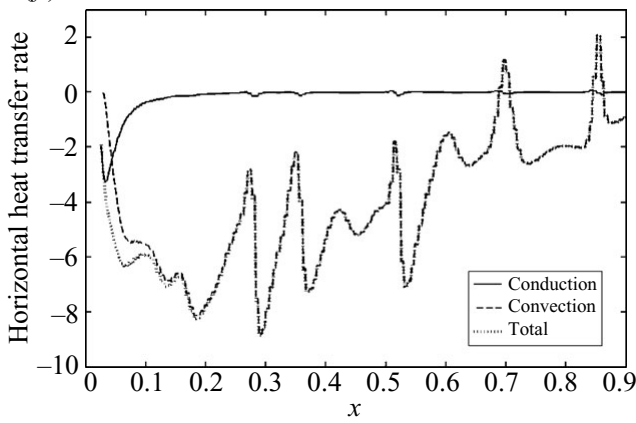

FIGURE 9. Flow properties at the quasi-steady state in the unstable convective regime of $R a>R a_{c}^{3}$. (a) Isotherms at an interval of 0.0042 for $R a=3.5 \times 10^{10}$. (b) Isotherms at an interval of 0.0017 for $R a=3.5 \times 10^{11}$. The interval of the isotherms in the enlarged region of both $(a)$ and $(b)$ is 0.0042 . (c) Streamlines at an interval of 7 for $R a=3.5 \times 10^{10}$. (d) Streamlines at an interval of 14 for $R a=3.5 \times 10^{11}$. Solid streamlines represent anticlockwise flow and dashed lines represent clockwise flow. $(e)$ and $(f)$ are profiles of the horizontal heat transfer rate averaged over the local depth for $R a=3.5 \times 10^{10}$ and $R a=3.5 \times 10^{11}$, respectively.

a certain threshold (0.002) were calculated from all of the simulation results and plotted against the scaling predictions $\left(x_{1} \sim L R a_{c}^{3 / 4} R a^{-1 / 4}\right)$ in figure $10(b)$. The good linear correlation clearly confirms the validity of the scaling. It is also noticeable that the dividing positions from the simulations shown in figure $10(b)$ correspond well with the horizontal positions for the origination of the wavy features suggested by figures $9(e)$ and $9(f)$ for the relevant Rayleigh numbers, confirming the consistence of the prediction of the extent of the unstable region from the perspectives of time and space. 
(a)

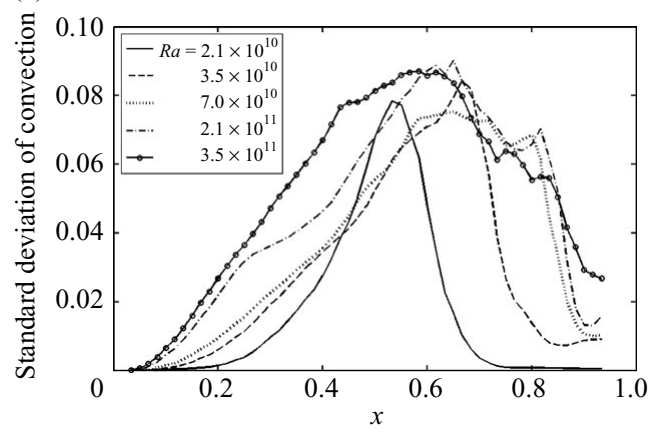

(b)

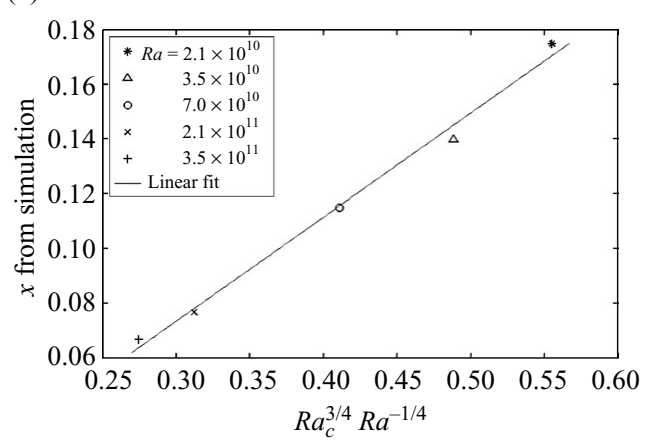

FIgURE 10. (a) Standard deviations of time series of the horizontal convection over a time period of 0.0012 for different Rayleigh numbers. $(b)$ Dividing positions between the stable and the unstable regions from numerical simulations versus scaling predictions.

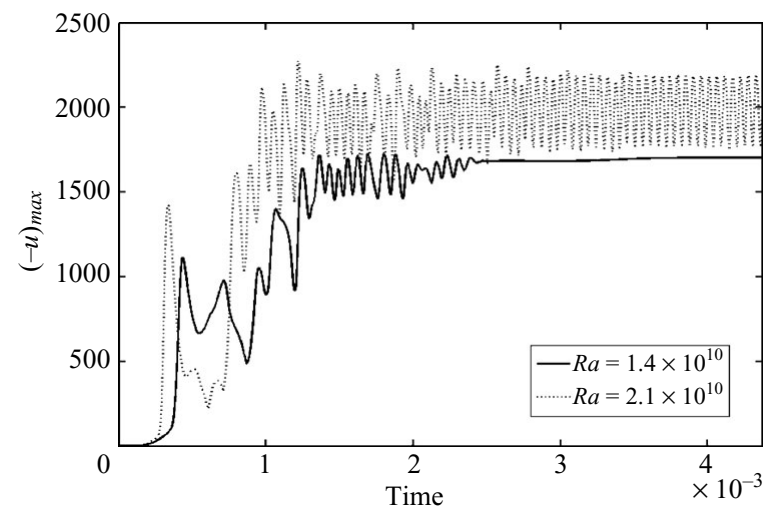

FIGURE 11. Time series of the maximum negative horizontal velocity at $x=0.35$ for two different Rayleigh numbers.

Among the simulation results for all the six Rayleigh numbers in the unstable regime as shown in figure 5 and table 1 , the result of the minimum Rayleigh number $R a=1.4 \times 10^{10}$ is different from the results of the other Rayleigh numbers in that, the flow for $R a=1.4 \times 10^{10}$ is unstable only for a certain period of time (the transitional stage), and finally approaches a steady state, whereas instability for the other Rayleigh numbers is maintained, and the flow remains quasi-steady. Figure 11 illustrates the above point with the time series of the maximum negative horizontal velocity at $x=0.35$ for $R a=1.4 \times 10^{10}$ and $R a=2.1 \times 10^{10}$ respectively. In Lei \& Patterson (2005), a similar trend of transition from unstable to steady-state flow was observed based on the mean flow rate (to be defined in (36)) over the entire domain for a Rayleigh number of $R a_{h}=7 \times 10^{5}$ corresponding to $R a=7 \times 10^{9}$. This transition from unstable to steady-state flow is consistent with the theoretical analysis of Forster (1971), which is based on the assumption of an infinite Prandtl number fluid subject to a constant heat flux from an upper horizontal boundary and an insulated horizontal bottom. Given the difference in the geometric configurations and the Prandtl numbers in Forster (1971) and the present investigation, our numerical results are consistent with the previous findings in that, for $R a_{h}>O\left(10^{7}\right)$ corresponding to $R a>O\left(10^{11}\right)$, the flow is fully intermittent whereas for relatively smaller Rayleigh numbers the 
flow eventually settles to a steady state. Full three-dimensional simulations are worth conducting in future investigations to reveal more detailed features of instability for the unstable flow regime.

\subsection{Steady-state scaling for distinct regions}

Two sets of scaling have been derived from the scaling analysis in $\S 3.2$ : one for the conduction-dominated region ((18)-(21)) and the other for stable-convectiondominated region $((22)-(25))$. In addition to the flow velocity, another flow property, i.e. the volumetric flow rate $Q(x)$ characterizing the intensity of the flow, is calculated from the simulation results for validating the scaling predication:

$$
Q(x)=1 / 2 \int_{-A x}^{0}|u| \mathrm{d} y .
$$

\subsubsection{Conduction-dominated region}

For the conduction-dominated region, scaling (18)-(21) applies. The buoyancy flow within the domain consists of two opposing directions: onshore along the water surface, and offshore along the sloping bottom. It can be derived from mass conservation that the scaling applies to both the surface and the bottom layers (Lei \& Patterson 2002). To validate the velocity scaling, the volumetric flow rate and the maximum velocities along the local depths at different offshore distances for different Rayleigh numbers from the simulation results are plotted against the scaling predictions in figure 12. It is clear that the volumetric flow rate and the velocity induced by surface cooling for conduction-dominated region are well represented by the scaling. Comparison between figures $12(b)$ and $12(c)$ shows that the slope of the linear fit for the surface layer is larger than that for the bottom layer, indicating the effect of different momentum boundary condition (stress free for the former and rigid non-slip for the latter). Compared to the stress-free boundary condition, the rigid non-slip boundary condition evidently limits the magnitude of the maximum flow velocity.

\subsubsection{Stable-convection-dominated region}

Similarly, for stable convection-dominated region, flow properties extracted from the simulations are plotted against the scaling predictions in figure 13. In the unstable flow regime, as indicated by figures $9(e)$ and $9(f)$, the region dominated by stable convection is very narrow. Therefore, only simulation results from the stable flow regime are used to validate the scaling for the stable-convection-dominated region. The evident linear relation in figure 13 validates the dependency of flow properties on $x$ and $R a$ predicted by scaling.

\subsubsection{Unstable region}

For the quasi-steady state flow in the unstable region, the time series of flow properties can be decomposed into a time-averaged mean value caused by the primary convection and a fluctuating component associated with flow instability. In the timeaveraged flow, the flow fluctuations are smoothed out as shown in figure 14 and the unstable region can be regarded as an extension of the stable-convection region. Therefore, the scaling for the stable-convection region is expected to hold true for the time-averaged mean flow in the unstable region as well. This hypothesis is validated in figure 15 with a linear correlation between the scaling and the simulation results. The same correlation between unstable regions and stable-convective regions of radiationinduced natural convection is validated in Mao et al. (2009b). 

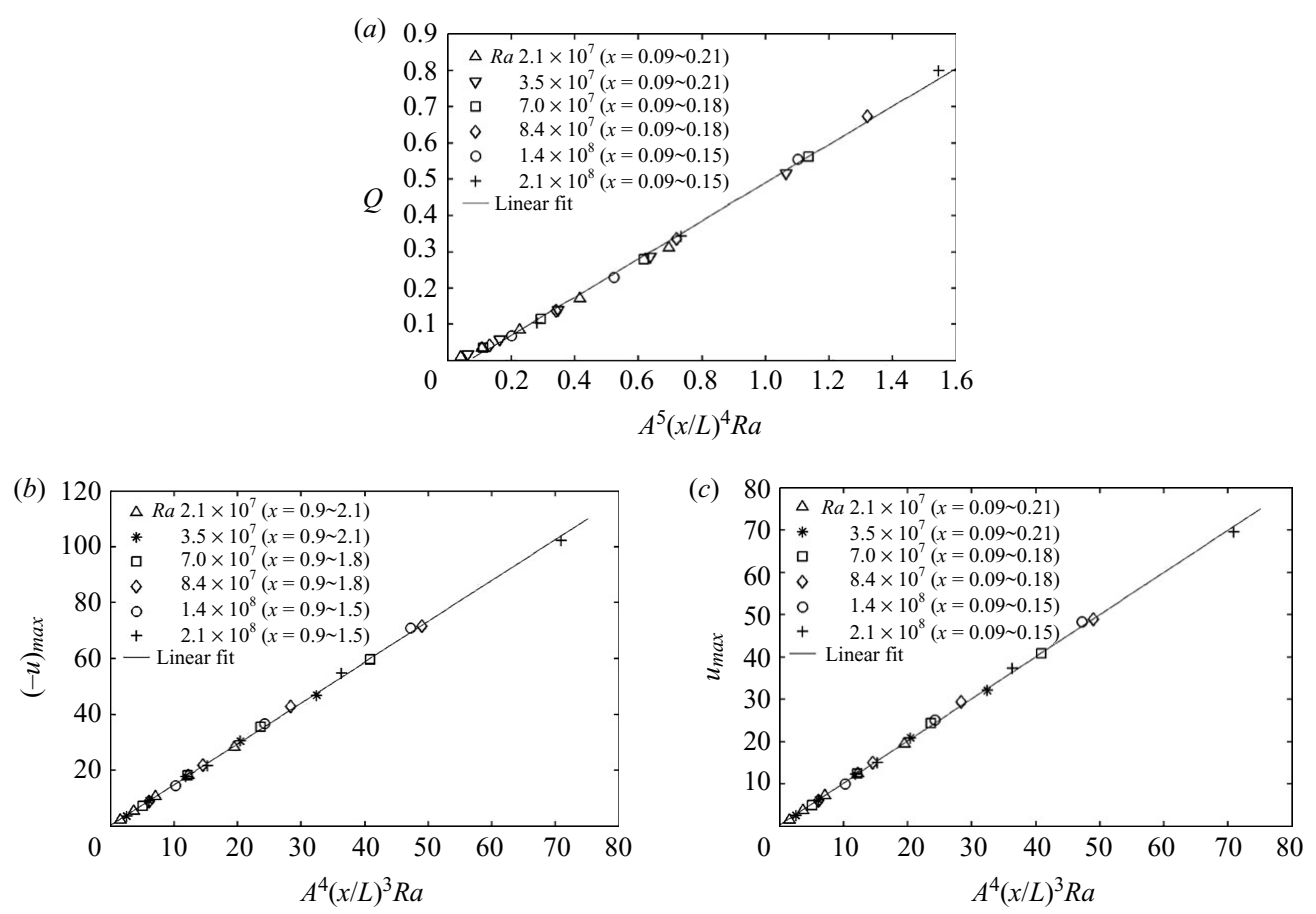

FIGURE 12. Volumetric flow rates and the maximum velocities extracted along vertical lines at various $x$ positions from simulation results versus their respective scaling for the conduction-dominated region. (a) Volumetric flow rates. $(b)$ The maximum negative horizontal velocities in the surface layer. (c) The maximum horizontal velocities in the bottom layer.

\section{Conclusions}

By introducing a variable offshore distance scale $x$, two critical functions for the Rayleigh number have been derived from scaling to identify the local flow features at $x: f_{1}(x) \sim A^{-6}(L / x)^{4}$ and $f_{2}(x) \sim R a_{c}^{3}(L / x)^{4}$. The thermal boundary layer is indistinct if $R a<f_{1}(x)$ and unstable if $R a>f_{2}(x)$. Comparison of these two functions reveals two possible scenarios depending on the bottom slope. The scenario for relatively large bottom slopes $A>R a_{c}^{-1 / 2}$ can be further classified into three flow regimes, which are examined in detail in this study. For each flow regime, distinctive subregions were identified by scaling and verified by numerical simulations. The major features of each flow regime are summarized below:

(i) For the conductive regime of $R a<A^{-6}$, the flow remains stable; and conduction dominates the horizontal heat transfer over the entire domain. At steady state, nearly vertical isotherms are distributed over the entire domain.

(ii) For the stable convective regime of $A^{-6}<R a<R a_{c}^{3}$, there are two distinct subregions separated at $x_{0} \sim A^{-3 / 2} \mathrm{Ra}^{-1 / 4} \mathrm{~L}$ : a conduction-dominated near-shore region and an offshore region dominated by stable convection.

(iii) For the unstable convective regime of $R a>R a_{c}^{3}$, there are three distinct subregions separated at $x_{0} \sim A^{-3 / 2} R a^{-1 / 4} L$ and $x_{1} \sim R a_{c}^{3 / 4} R a^{-1 / 4} L$ : a near-shore region dominated by conduction, a central region dominated by stable convection and an offshore region dominated by unstable convection.

Two different sets of scaling relations have been derived for the steady-state flow of the conduction-dominated region (scales (18)-(21)) and the region dominated by 

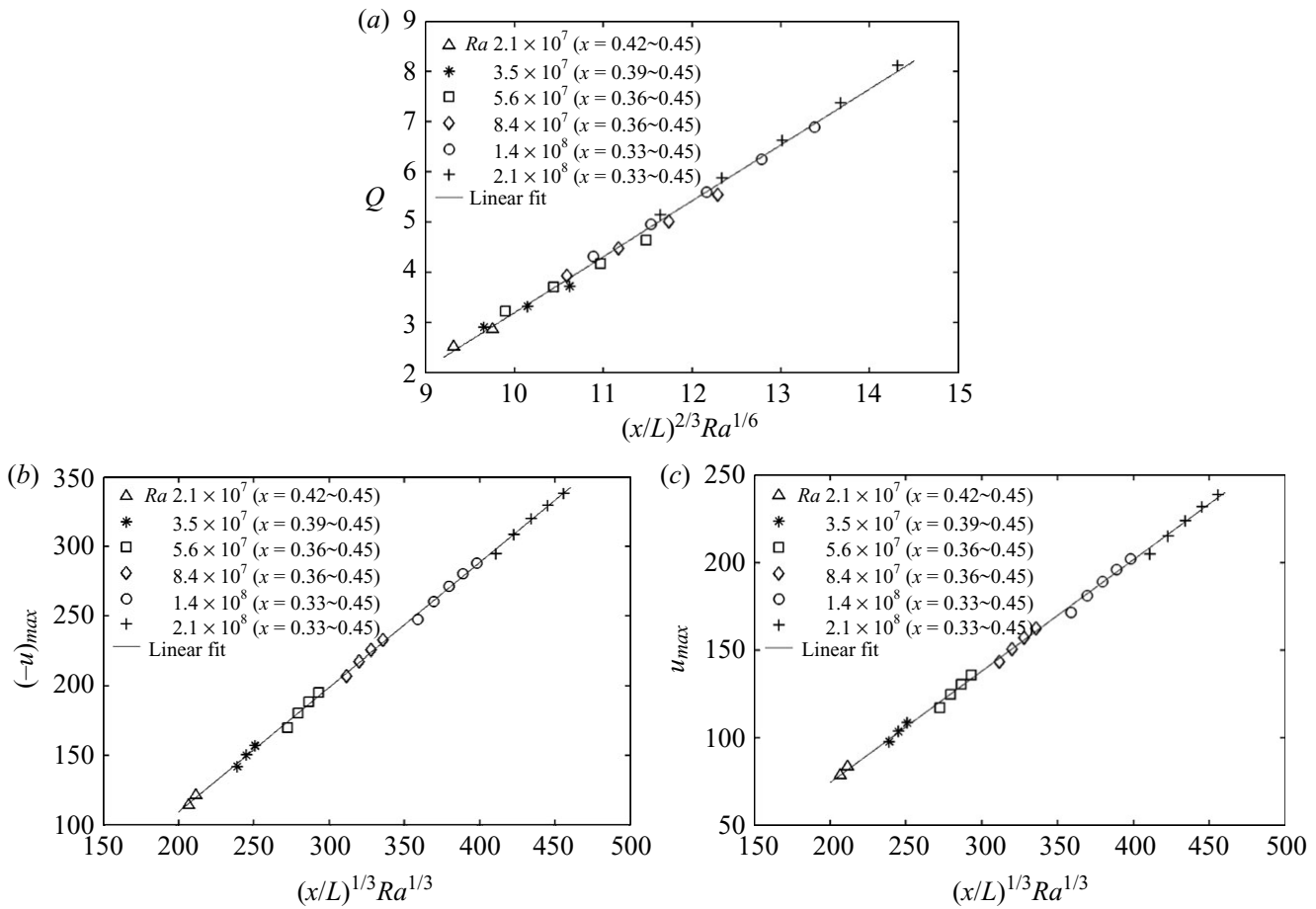

FiguRE 13. Volumetric flow rates and the maximum velocities extracted along vertical lines at various $x$ positions from simulation results versus their respective scaling for stable-convection-dominated region. (a) Volumetric flow rates. (b) The maximum negative horizontal velocities in the surface layer. $(c)$ The maximum horizontal velocities in the bottom layer.

(a)

(b)

FiguRE 14. Contours of the time-averaged flow over a period of 0.0012 during the quasi-steady state for $R a=2.1 \times 10^{10}$. (a) Streamlines at an interval of 10 , dashed lines represent clockwise flow and solid lines represent anticlockwise flow. (b) Isotherms at an interval of 0.007 .

stable-convection (scales (22)-(25)) respectively. For the unstable flow regime, it is revealed that the time-averaged mean flow of the unstable region in quasi-steady state is scaled the same as the region dominated by stable convection.

The results of the scaling analysis are readily applicable to experimental designs and estimations of the significance of this cooling-induced flow in field situations, as they provide detailed estimation of the flow properties and the distinctive subregions for different flow regimes. Similar to the case with radiative heating, in a real life situation, the intensity of the surface cooling usually places the flow in regime (iii) above, indicating that the unstable region always exists in a region offshore, the extent 

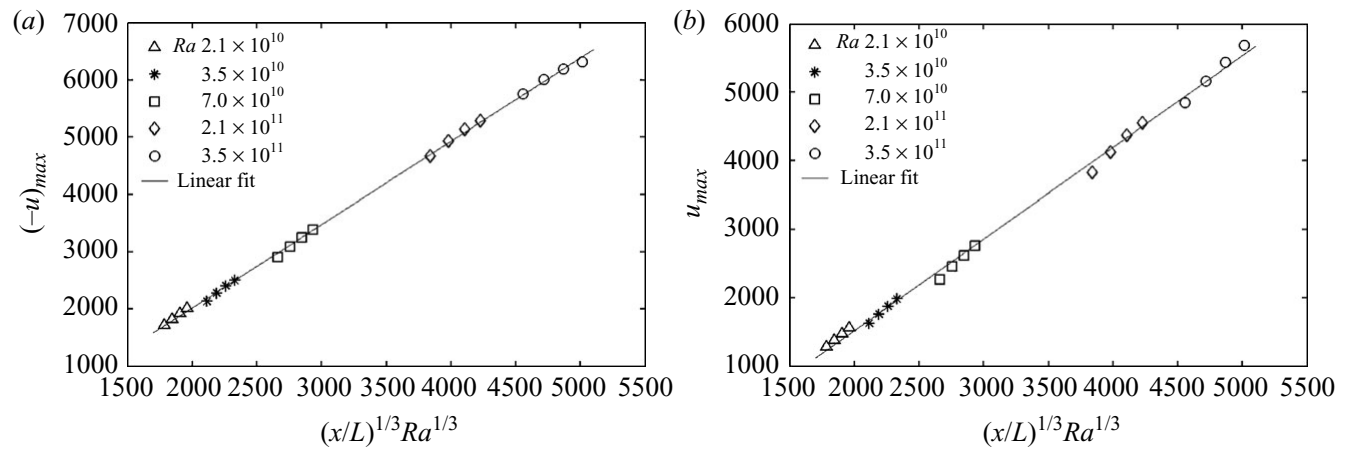

FIGURE 15. The maximum velocities extracted along vertical lines at different horizontal positions within the unstable region $(x=0.27 \sim 0.36$ at an equal interval for all the Rayleigh numbers) for the time-averaged mean flow. (a) The maximum negative horizontal velocity in the surface layer $(b)$ The maximum horizontal velocity in the bottom layer.

of which is provided by the present scaling. The different flow scenarios, flow regimes and distinctive subregions revealed by the present scaling provide important insights into the cooling-induced exchange flows at various offshore distances, which have significant biological and environmental implications as detailed in the introduction. Together with the recent findings for the radiation-induced natural convection (Mao et al. 2009a,b), a more thorough understanding of near-shore natural convection flow driven by the idealized constant thermal forcing has been achieved. While a steady or quasi-steady state will finally be reached for constant thermal forcing, in real field situations, the diurnal variation of the thermal forcing and the inertia of the flow result in an unsteady flow and a time lag between the flow response and the thermal forcing as demonstrated by field experiments (Adams \& Wells 1984; Monismith et al. 1990). Therefore, further understanding of the exchange flows relevant to field conditions entails future investigations of the 'thermal siphon' under time-varying thermal forcing.

As mentioned in Mao et al. (2009a), in application of the scaling to field situations, many other geophysical factors must be accounted for: the rotation of the earth at large scales, the variation of the geometry and the interaction of the thermal flow with currents driven by other mechanisms (e.g. tides, waves, wind-driven currents, geostrophic currents). Each of these is, in its own right, an important mechanism. A study of each, such as the present case, in isolation allows development of a more comprehensive understanding of the overall response of the system.

The authors gratefully acknowledge the financial support of the Australian Research Council. This work was carried out while Yadan Mao was a recipient of a $\mathrm{PhD}$ scholarship jointly awarded by China Scholarship Council and James Cook University.

\section{REFERENCES}

Adams, E. E. \& Wells, S. A. 1984 Field measurements on side arms of lake. J Hydraul. Engng ASLE 110, 773-793.

Bednarz, T. P., Lei, C. \& Patterson, J. C. 2008 An experimental study of unsteady natural convection in a reservoir model cooled from the water surface. Exp. Therm. Fluid Sci. 32, 844-856.

Bednarz, T. P., Lei, C. \& Patterson, J. C. 2009 A numerical study of unsteady natural convection induced by iso-flux surface cooling in a reservoir model. Intl J. Heat Mass Transfer 52, 56-66. 
Drazin, P. G. \& ReID, W. H. 1981 Hydrodynamic Stability. Cambridge University Press.

Forster, T. D. 1971 Intermittent convection. Geophys. Fluid Dyn. 2, 201-217.

FArrow, D. E. \& Patterson, J. C. 1993a On the response of a reservoir sidearm to diurnal heating and cooling. J. Fluid Mech. 246, 143-161.

Farrow, D. E. \& Patterson, J. C. $1993 b$ On the stability of the near shore waters of a lake when subject to solar heating. Intl J. Heat Mass Transfer 36, 89-100.

Farrow, D. E. \& PAtTerson, J. C. 1994 The daytime circulation and temperature structure in a reservoir sidearm. Intl J. Heat Mass Transfer 37, 1954-1968.

Horsch, G. M. \& Stefan, H. G. 1988 Convective circulation in littoral water due to surface cooling. Limnol. Oceanogr. 33, 1068-1083.

Hughes, G. O. \& Griffiths, R. W. 2008 Horizontal convection. Annu. Rev. Fluid Mech. 40, 185-208.

Lei, C. \& Patterson, J. C. 2002 Unsteady natural convection in a triangular enclosure induced by absorption of radiation. J. Fluid Mech. 460, 181-209.

Lei, C. \& Patterson, J. C. 2005 Unsteady natural convection in a triangular enclosure induced by surface cooling. Intl J. Heat Fluid Flow 26, 307-321.

Mao, Y., Lei, C. \& Patterson, J. C. 2007 Natural convection in a triangular enclosure induced by solar radiation. Proceedings of the Sixteenth Australasia Fluid Mechanics Conference, Gold Coast, Australia, pp. 406-410.

Mao, Y., Lei, C. \& Patterson, J. C. $2009 a$ Unsteady natural convection in a triangular enclosure induced by absorption of radiation - a revisit by improved scaling analysis. J. Fluid Mech. 622, 75-102.

Mao, Y., Lei, C. \& Patterson, J. C. $2009 \mathrm{~b}$ Characteristics of instability of radiationinduced natural convection in shallow littoral waters. Intl J. Thermal Sci., doi:10.1016/ j.ijthermalsci.2009.07.004 (in press).

Monismith, S. G., Imberger, J. \& Morison, M. L. 1990 Convective motions in the sidearm of a small reservoir. Limnol. Oceanogr. 35, 1676-1702.

Monismith, S. G., Genin, A., Reidenbach, M. A., Yahel, G. \& Koseff, J. R. 2006 Thermally driven exchanges between a coral reef and the adjoining ocean. J. Phys. Oceanogr. 36, 1332-1347.

Monismith, S. G. 2007 Hydrodynamics of coral reefs. Annu. Rev. Fluid Mech. 39, 37-55.

Mullarney, J. C., Griffiths, R. W. \& Hughes, G. O. 2004 Convection driven by differential heating at a horizontal boundary. J. Fluid Mech. 516, 181-209.

Niemann, H., Richter, C., Jonkers, H. M. \& Badran, M. I. 2004 Red Sea gravity currents cascade near-reef phytoplankton to the twilight zone. Mar. Ecol. Prog. Ser. 269, 91-99.

Phillips, O. M. 1970 On flows induced by diffusion in a stably stratified fluid. Deep-Sea Res. 17, 435-443.

Rossby, H. T. 1965 On thermal convection driven by non-uniform heating from below. Deep-Sea Res. 12, 9-16.

Sturman, J. J., Oldham, C. E. \& Ivey, G. N. 1999 Steady convective exchange flows down slopes. Aqua. Sci. 61, 260-278.

WunsCH, C. 1970 On oceanic boundary mixing. Deep-Sea Res. 17, 293-301. 